\title{
A Recursive Elimination Method for Finite-Horizon Optimal Control Problems of Discrete-Time Rational Systems
}

\author{
AUTHOR(S):
}

Ohtsuka, Toshiyuki

\section{CITATION:}

Ohtsuka, Toshiyuki. A Recursive Elimination Method for Finite-Horizon Optimal Control Problems of Discrete-Time Rational Systems. IEEE Transactions on Automatic Control 2014, 59(11): 3081-3086

ISSUE DATE:

2014-11

URL:

http://hdl.handle.net/2433/191217

\section{RIGHT:}

(c) 2014 IEEE. Personal use of this material is permitted. Permission from IEEE must be obtained for all other uses, in any current or future media, including reprinting/republishing this material for advertising or promotional purposes, creating new collective works, for resale or redistribution to servers or lists, or reuse of any copyrighted component of this work in other works.; この論文は出版社版でありません。引用の際には出版社版をご確認ご利用ください。; This is not the published version. Please cite only the published version. 


\section{A Recursive Elimination Method for Finite-Horizon Optimal Control Problems of Discrete-Time Rational Systems}

\author{
Toshiyuki Ohtsuka, Member IEEE
}

\begin{abstract}
In this technical note, a method of solving finite-horizon optimal control problems involving discrete-time rational systems is proposed. Sequences of algebraic equations for the control input and costate at each time are constructed backward, starting from the terminal condition, by the recursive elimination of variables in the optimality conditions. This recursive elimination can be viewed as a generalization of the classical backward sweep method to obtain the discretetime Riccati equation for finite-horizon linear quadratic control. Sufficient conditions are given for the existence and uniqueness of locally optimal state feedback laws in the form of algebraic functions of the state.
\end{abstract}

Index Terms - optimal control, nonlinear systems, discrete-time systems, Euler-Lagrange equations

\section{INTRODUCTION}

Optimal control has been one of the most important problems in systems and control theory. Classical and successful results in optimal control include linear quadratic (LQ) control [1], which can be solved by means of the Riccati equation. In general, optimal control problems for nonlinear systems cannot be solved explicitly, and various numerical methods have been proposed for solving them in the literature.

In this technical note, as an alternative to the conventional numerical methods, an algebraic method is proposed for solving finite-horizon optimal control problems (FHOCPs) of discrete-time rational systems by extending preliminary results for polynomial systems [2]. First, the discrete-time Euler-Lagrange equations (ELE), which essentially correspond to the Karush-Kuhn-Tucker (KKT) conditions [3], are recast as a set of algebraic equations consisting of only polynomials. Then, a systematic method of constructing a sequence of algebraic equations with smaller sizes is proposed so that the unknowns (the costate and control input) at each time can be obtained by solving an algebraic equation involving only the variables at that time. Since the sequence of algebraic equations for the costate is obtained recursively starting from the terminal condition in the ELE, the proposed method can be regarded as a generalization of the classical backward sweep method [4], in which the discrete-time Riccati equation is solved backward to obtain the costate as a function of the state.

Once the algebraic equations are obtained so as to determine the unknowns as implicit functions of the state

This work has been partly supported by a Grant-in-Aid for Exploratory Research (No. 24656263) from Japan Society for the Promotion of Science.

T. Ohtsuka is with the Department of Systems Science, Graduate School of Informatics, Kyoto University, Yoshida-Honmachi, Sakyoku, Kyoto 606-8501, Japan (e-mail: ohtsuka@i.kyoto-u.ac.jp). at each time, they can be solved by any numerical method such as by using Newton's method pointwise for each state. Therefore, the solutions need not be stored over a domain in the state space. In particular, only the algebraic equation for the control input at each time needs to be solved to realize state feedback control.

In contrast to related approaches [5]-[10] in the literature, the present approach deals with FHOCPs for discrete-time rational systems. Moreover, the proposed method is not a direct application of algebraic tools such as Gröbner bases because the particular structure of the optimal control problem is exploited to recursively construct the algebraic equations for the costates and control inputs (for applications of Gröbner bases in control design, see, for example, [11]). Sufficient conditions are also given for the existence and uniqueness of locally optimal state feedback laws in the form of algebraic functions of the state.

Notation: A suffix $k$ denotes the time in discrete-time systems throughout this technical note, while $i$ and $j$ denote the components of a vector. For example, $x_{k}$ denotes a vector at time $k$, while $x_{i}$ denotes the $i$ th component of a vector $x$. Similarly, $x_{k i}$ denotes the $i$ th component of a vector $x_{k}$. To avoid confusion in suffixes, $x_{k i}$ is also denoted by $x_{k, i}$ when necessary. For a field $K$ and vectors $X=\left[\begin{array}{lll}X_{1} & \cdots & X_{n}\end{array}\right]^{\mathrm{T}}$ and $Y=\left[\begin{array}{lll}Y_{1} & \cdots & Y_{m}\end{array}\right]^{\mathrm{T}}, K[X, Y]$ and $K(X, Y)$ denote the ring of polynomials and field of rational functions, respectively, in the components of $X$ and $Y$ over $K$. The same symbols are often used to denote an indeterminate as well as an element of a certain set. An ideal generated by $g_{1}, \ldots, g_{\ell} \in K[X]$ is denoted by $\left\langle g_{1}, \ldots, g_{\ell}\right\rangle$. That is, $\left\langle g_{1}, \ldots, g_{\ell}\right\rangle:=\left\{s_{1} g_{1}+\cdots+s_{\ell} g_{\ell}\right.$ : $\left.s_{1}, \ldots, s_{\ell} \in K[X]\right\}$. An ideal generated by the components of vectors $G_{i} \in K[X]^{\nu_{i}}\left(i=1, \ldots, \ell ; \nu_{i} \in \mathbf{N}\right)$ is also simply denoted by $\left\langle G_{1}, \ldots, G_{\nu}\right\rangle$ for brevity. For a field $K$, $\bar{K}$ denotes the algebraic closure of $K$. For an ideal $I$ of a polynomial ring $K[X]$ with $X=\left[\begin{array}{lll}X_{1} & \cdots & X_{n}\end{array}\right]^{\mathrm{T}}$, the affine variety defined by $I$ is the set of elements (points) in $\bar{K}^{n}$ where all elements of $I$ vanish and is denoted by $\mathcal{V}(I)$. For $I=\left\langle g_{1}, \ldots, g_{\ell}\right\rangle \subset K[X], \mathcal{V}(I)$ is the set of common zeros of $g_{1}, \ldots, g_{\ell}$. The restriction of $\mathcal{V}(I)$ into $K^{n}$ is denoted by $\mathcal{V}_{K}(I)$, i.e., $\mathcal{V}_{K}(I):=\mathcal{V}(I) \cap K^{n}$. For a scalar-valued function $V(x)$, we denote a row vector consisting of the partial derivatives of $V$ with respect to $x_{i}(i=1, \ldots, n)$ as $\partial V / \partial x$, and the column vector $(\partial V / \partial x)^{\mathrm{T}}$, which is the transpose of $\partial V / \partial x$, as $\nabla_{x} V$. For simple notation, a suffix $k$ is often omitted, such as in the use of $\nabla_{x} V\left(x_{k}\right)$ to denote $\nabla_{x_{k}} V\left(x_{k}\right)$.

\section{Problem Formulation}

We consider the discrete-time system

$$
x_{k+1}=f_{k}\left(x_{k}, u_{k}\right),
$$

where $x_{k} \in \mathbf{R}^{n}$ and $u_{k} \in \mathbf{R}^{m}$ respectively denote the state and input for the system, and the initial state $x_{0}$ is given. The vector-valued function $f_{k}$ is assumed to be a rational function of $x_{k}$ and $u_{k}$ for all $k=0, \cdots, N-1$ with $N$ a 
positive integer. The performance index to be minimized is given as

$$
J=\varphi\left(x_{N}\right)+\sum_{k=0}^{N-1} L_{k}\left(x_{k}, u_{k}\right)
$$

where $\varphi$ and $L_{k}$ are scalar-valued functions. In this technical note, we assume that $\nabla_{x} \varphi$ consists of algebraic functions and $\nabla_{x} L_{k}$ and $\nabla_{u} L_{k}$ are assumed to be rational functions. The notion of algebraic functions is formally defined as follows.

Definition 1: An analytic function $\rho: U \rightarrow \mathbf{R}$ defined on an open set $U \subset \mathbf{R}^{n}$ is said to be an algebraic function if there exists a nonzero polynomial $\Phi(x, X) \in \mathbf{R}(x)[X]$ such that $\Phi(x, \rho(x))=0$ holds for all $x \in U$.

Remark 1: By multiplying by some polynomial in $\mathbf{R}[x]$, $\Phi(x, X)$ can always be chosen from $\mathbf{R}[x, X]$. For simple notation, we regard an algebraic function $\rho$ as an element of $\overline{\mathbf{R}(x)}$, the algebraic closure of $\mathbf{R}(x)$, and write $\rho \in \overline{\mathbf{R}(x)}$ irrespective of its domain of definition.

It is readily shown that the set of equality constraints given by (1) for $k=0, \ldots, N-1$ satisfy the linear independence constraint qualification [3] for any feasible sequence of states and inputs. Then, there exist Lagrange multipliers satisfying the KKT conditions, i.e., the necessary conditions for optimality, at an optimal solution. Owing to the particular structure of the optimal control problem, the KKT conditions lead to the following discrete-time ELE for $k=0, \ldots, N-1$ :

$$
\begin{aligned}
& x_{k+1}=f_{k}\left(x_{k}, u_{k}\right), \\
& p_{k}=\nabla_{x} H_{k}\left(x_{k}, u_{k}, p_{k+1}\right), \\
& p_{N}=\nabla_{x} \varphi\left(x_{N}\right), \\
& \nabla_{u} H_{k}\left(x_{k}, u_{k}, p_{k+1}\right)=0,
\end{aligned}
$$

where $p_{k}$ is the Lagrange multiplier, called the costate (or adjoint variable), and $H_{k}$ denotes the Hamiltonian defined as

$$
H_{k}\left(x_{k}, u_{k}, p_{k+1}\right)=L_{k}\left(x_{k}, u_{k}\right)+p_{k+1}^{\mathrm{T}} f_{k}\left(x_{k}, u_{k}\right) .
$$

The ELE (2) - (5) can be viewed as a two-point boundaryvalue problem for sequences of states and costates, because the initial state $x_{0}$ is specified while the terminal condition is imposed on $p_{N}$.

In the present setting, $\nabla_{x} \varphi\left(x_{N}\right)$ consists of algebraic functions, and all other functions are rational functions in the ELE. Therefore, the ELE can be rewritten as a set of polynomial equations as described below. First, since each component of $\nabla_{x} \varphi\left(x_{N}\right)$ is an algebraic function, there exists $F_{N} \in \mathbf{R}\left[x_{N}, p_{N}\right]^{n}$ such that

$$
F_{N}\left(x_{N}, \nabla_{x} \varphi\left(x_{N}\right)\right)=0
$$

holds for all $x_{N}$ in an open subset of $\mathbf{R}^{n}$ where $\nabla_{x} \varphi\left(x_{N}\right)$ is defined. Next, other rational functions are expressed as fractions of polynomials as

$$
\begin{aligned}
f_{k}\left(x_{k}, u_{k}\right) & =D_{x k}^{-1}\left(x_{k}, u_{k}\right) n_{x k}\left(x_{k}, u_{k}\right), \\
D_{x k} & =\operatorname{diag}\left[d_{x k i}\right] \in \mathbf{R}\left[x_{k}, u_{k}\right]^{n \times n}, \\
n_{x k} & \in \mathbf{R}\left[x_{k}, u_{k}\right]^{n}, \\
\nabla_{x} H_{k}\left(x_{k}, u_{k}, p_{k+1}\right) & =D_{p k}^{-1}\left(x_{k}, u_{k}\right) n_{p k}\left(x_{k}, u_{k}, p_{k+1}\right), \\
D_{p k} & =\operatorname{diag}\left[d_{p k i}\right] \in \mathbf{R}\left[x_{k}, u_{k}\right]^{n \times n}, \\
n_{p k} & \in \mathbf{R}\left[x_{k}, u_{k}, p_{k+1}\right]^{n}, \\
\nabla_{u} H_{k}\left(x_{k}, u_{k}, p_{k+1}\right) & =D_{u k}^{-1}\left(x_{k}, u_{k}\right) n_{u k}\left(x_{k}, u_{k}, p_{k+1}\right), \\
D_{u k} & =\operatorname{diag}\left[d_{u k i}\right] \in \mathbf{R}\left[x_{k}, u_{k}\right]^{m \times m}, \\
n_{u k} & \in \mathbf{R}\left[x_{k}, u_{k}, p_{k+1}\right]^{m},
\end{aligned}
$$

where all pairs consisting of a numerator and denominator are chosen to be coprime to each other. Then, the ELE are rewritten as

$$
\begin{aligned}
& D_{x k}\left(x_{k}, u_{k}\right) x_{k+1}-n_{x k}\left(x_{k}, u_{k}\right)=0 \\
& D_{p k}\left(x_{k}, u_{k}\right) p_{k}-n_{p k}\left(x_{k}, u_{k}, p_{k+1}\right)=0 \\
& F_{N}\left(x_{N}, p_{N}\right)=0 \\
& n_{u k}\left(x_{k}, u_{k}, p_{k+1}\right)=0 \\
& \bar{d}_{k}\left(x_{k}, u_{k}\right):=\prod_{i=1}^{n} d_{x k i}\left(x_{k}, u_{k}\right) \prod_{i=1}^{n} d_{p k i}\left(x_{k}, u_{k}\right) \\
& \quad \times \prod_{i=1}^{m} d_{u k i}\left(x_{k}, u_{k}\right) \neq 0
\end{aligned}
$$

where the last condition (10) is imposed so that no denominator polynomial in the ELE vanishes. Repeated factors in $\bar{d}_{k}$ can be omitted as long as the zeros of $\bar{d}_{k}$ are unchanged. Moreover, the last condition (10) can also be expressed as an equality by introducing a new scalar variable $y_{k}$ as

$$
1-y_{k} \bar{d}_{k}\left(x_{k}, u_{k}\right)=0
$$

which can be satisfied by some $y_{k} \in \mathbf{R}$ if and only if $\bar{d}_{k}\left(x_{k}, u_{k}\right) \neq 0$. Now all functions in (6)-(11) are polynomials, to which tools in commutative algebra and algebraic geometry are applicable. The initial state $x_{0}$ will be regarded as a parameter hereafter so as to characterize a family of solutions, rather than a particular solution, of the ELE.

\section{Elimination Method}

If the ELE (2) - (5), or equivalently (6)-(11), are decomposed into a set of independent algebraic equations for the state, costate, and control input at each time, then each set of algebraic equations may determine the costate and control input as implicit functions of the state at each time. The decomposition of the ELE into a set of independent algebraic equations can be viewed as the elimination of variables. For the systematic elimination of variables in the ELE, some tools from commutative algebra and algebraic geometry [12], [13] are introduced.

For an ideal $I=\left\langle g_{1}, \ldots, g_{\ell}\right\rangle \subset \mathbf{R}[X, Y]$ with $X=$ $\left[\begin{array}{lll}X_{1} & \cdots & X_{n}\end{array}\right]^{\mathrm{T}}$ and $Y=\left[\begin{array}{llll}Y_{1} & \cdots & Y_{m}\end{array}\right]^{\mathrm{T}}, \mathcal{V}_{\mathbf{R}}(I) \subset \mathbf{R}^{n} \times \mathbf{R}^{m}$ represents the set of real-valued solutions to the algebraic equations $g_{1}(X, Y)=0, \ldots, g_{\ell}(X, Y)=0$. Note that every 
polynomial in $I$ vanishes at any $(X, Y) \in \mathcal{V}_{\mathbf{R}}(I)$. If a polynomial $h \in \mathbf{R}[Y]$ also belongs to $I$, then $h(Y)=0$ holds for any $(X, Y) \in \mathcal{V}_{\mathbf{R}}(I)$. That is, $I \cap \mathbf{R}[Y]$ gives the set of polynomials that vanish at the $Y$-coordinates of points in $\mathcal{V}_{\mathbf{R}}(I)$. More precisely, the affine variety containing all the $Y$-coordinates of points in $\mathcal{V}_{\mathbf{R}}(I)$ is characterized by the following lemma [13].

Lemma 1: For an ideal $I \in \mathbf{R}[X, Y]$, the following holds:

$$
\pi_{Y}\left(\mathcal{V}_{\mathbf{R}}(I)\right) \subset \mathcal{V}_{\mathbf{R}}(I \cap \mathbf{R}[Y]),
$$

where $\pi_{Y}\left(\mathcal{V}_{\mathbf{R}}(I)\right)$ denotes the projection of $\mathcal{V}_{\mathbf{R}}(I)$ on the $Y$-space, i.e.,

$\pi_{Y}\left(\mathcal{V}_{\mathbf{R}}(I)\right):=\left\{Y \in \mathbf{R}^{m}:(X, Y) \in \mathcal{V}_{\mathbf{R}}(I)\right.$ for some $\left.X \in \mathbf{R}^{n}\right\}$. It is readily shown that $I \cap \mathbf{R}[Y]$ is an ideal, and $I \cap \mathbf{R}[Y]$ is called the elimination ideal of $I$ with respect to $Y$.

A basis (a set of generators) of an elimination ideal can be computed by using a Gröbner basis [12], [13], which is a set of generators with some nice properties and whose computation algorithm is implemented in various symbolic computation systems. The following lemma [13] gives a computation method for an elimination ideal.

Lemma 2 (Elimination Theorem): Let $G_{B}$ be a Gröbner basis of an ideal $I \subset \mathbf{R}[X, Y]$ with respect to a lexicographic order such that $X_{1}>\cdots>X_{n}>Y_{1}>$ $\cdots>Y_{m}$. Then, $G_{B} \cap \mathbf{R}[Y]$ is a Gröbner basis of $I \cap \mathbf{R}[Y]$.

Note that any ideal of a polynomial ring over a field has a Gröbner basis consisting of a finite number of generators [12], [13]. It should also be noted that the number of generators can be specified for a particular class of ideals (see Lemma 4 in Section V).

By a simple application of Lemma 1 and Lemma 2, algebraic equations can be obtained for the state, costate, and control input at each time.

Algorithm 1: Define ideals $\bar{I}^{E L E}, I_{k}^{E L E}(k=0, \ldots, N)$, and $K_{k}^{E L E}(k=0, \ldots, N-1)$ as

$$
\begin{aligned}
\bar{I}^{E L E}:= & \left\langle D_{x 0} x_{1}-n_{x 0}, D_{p 0} p_{0}-n_{p 0}, n_{u 0}, 1-y_{0} \bar{d}_{0}, \ldots,\right. \\
& D_{x, N-1} x_{N}-n_{x, N-1}, D_{p, N-1} p_{N-1}-n_{p, N-1}, \\
& \left.n_{u, N-1}, 1-y_{N-1} \bar{d}_{N-1}, F_{N}\right\rangle \\
& \subset \mathbf{R}\left[x_{0}, p_{0}, u_{0}, y_{0}, \ldots, x_{N-1}, p_{N-1}, u_{N-1},\right. \\
& \left.y_{N-1}, x_{N}, p_{N}\right], \\
I_{k}^{E L E}:= & \bar{I}^{E L E} \cap \mathbf{R}\left[x_{k}, p_{k}\right], \\
K_{k}^{E L E}:= & \bar{I}^{E L E} \cap \mathbf{R}\left[x_{k}, u_{k}\right] .
\end{aligned}
$$

If sequences of the states $\left(x_{k}\right)_{k=0}^{N}$, costates $\left(p_{k}\right)_{k=0}^{N}$, and control inputs $\left(u_{k}\right)_{k=0}^{N-1}$ satisfy the ELE, Lemma 1 implies that $\left(x_{k}, p_{k}\right) \in \mathcal{V}_{\mathbf{R}}\left(I_{k}^{E L E}\right)(k=0, \ldots, N)$ and $\left(x_{k}, u_{k}\right) \in \mathcal{V}_{\mathbf{R}}\left(K_{k}^{E L E}\right)(k=0, \ldots, N-1)$ hold. Therefore, the generators of an ideal constructed by Algorithm 1 define an algebraic equation satisfied by any solution to the ELE, and this equation can be used to determine the costate or control input as an implicit function of the state. However, the maximal degree and cardinality of the generators of a Gröbner basis are known to be doubleexponential to the number of generators of a given ideal in the worst case [14]. Therefore, it is computationally preferable to compute a sequence of Gröbner bases of ideals with fewer generators than to construct the Gröbner basis for all the polynomials in the ELE.

\section{Recursive Elimination Method}

In the case of the LQ control problem, a sequence of optimal state feedback laws is obtained by recursively solving the discrete-time Riccati equation backward, which motivates the following algorithm for constructing algebraic equations for the costate and input recursively.

Algorithm 2 (Recursive Elimination Method):

1) Let $I_{N}:=\left\langle F_{N}\right\rangle \subset \mathbf{R}\left[x_{N}, p_{N}\right]$, and let $k:=N-1$.

2) Do while $k \geq 0$

a) Define an ideal $\bar{I}_{k} \subset \mathbf{R}\left[x_{k+1}, p_{k+1}, x_{k}, u_{k}, p_{k}\right.$, $\left.y_{k}\right]$ as

$$
\begin{aligned}
\bar{I}_{k}:= & \left\langle F_{k+1}, D_{x k} x_{k+1}-n_{x k}, D_{p k} p_{k}-n_{p k},\right. \\
& \left.n_{u k}, 1-y_{k} \bar{d}_{k}\right\rangle .
\end{aligned}
$$

b) Define ideals $I_{k} \subset \mathbf{R}\left[x_{k}, p_{k}\right]$ and $K_{k} \subset$ $\mathbf{R}\left[x_{k}, u_{k}\right]$ as

$$
\begin{aligned}
I_{k} & :=\left\langle F_{k}\right\rangle=\bar{I}_{k} \cap \mathbf{R}\left[x_{k}, p_{k}\right], \\
K_{k} & :=\left\langle G_{k}\right\rangle=\bar{I}_{k} \cap \mathbf{R}\left[x_{k}, u_{k}\right],
\end{aligned}
$$

where $F_{k}$ and $G_{k}$ are vectors of the generators of $\bar{I}_{k} \cap \mathbf{R}\left[x_{k}, p_{k}\right]$ and $\bar{I}_{k} \cap \mathbf{R}\left[x_{k}, u_{k}\right]$, respectively.

c) $k:=k-1$

Theorem 1: Suppose sequences of the states $\left(x_{k}\right)_{k=0}^{N}$, costates $\left(p_{k}\right)_{k=0}^{N}$, and control inputs $\left(u_{k}\right)_{k=0}^{N-1}$ satisfy the ELE. Then, for $I_{k}=\left\langle F_{k}\right\rangle(k=0, \ldots, N)$ and $K_{k}=\left\langle G_{k}\right\rangle$ $(k=0, \ldots, N-1)$ obtained by Algorithm $2,\left(x_{k}, p_{k}\right) \in$ $\mathcal{V}_{\mathbf{R}}\left(I_{k}\right)(k=0, \ldots, N)$ and $\left(x_{k}, u_{k}\right) \in \mathcal{V}_{\mathbf{R}}\left(K_{k}\right)(k=$ $0, \ldots, N-1)$ hold. That is, $F_{k}\left(x_{k}, p_{k}\right)=0(k=0, \ldots, N)$ and $G_{k}\left(x_{k}, u_{k}\right)=0(k=0, \ldots, N-1)$ hold.

Proof: Suppose sequences of the states $\left(x_{k}\right)_{k=0}^{N}$, costates $\left(p_{k}\right)_{k=0}^{N}$, and control inputs $\left(u_{k}\right)_{k=0}^{N-1}$ satisfy the ELE and, equivalently, (6)-(11). First, let $k=N$. Since $F_{N}\left(x_{N}, p_{N}\right)=0$ holds, $\left(x_{N}, p_{N}\right) \in \mathcal{V}_{\mathbf{R}}\left(I_{N}\right)$ holds as claimed. Next, suppose $\left(x_{k}, p_{k}\right) \in \mathcal{V}_{\mathbf{R}}\left(I_{k}\right)$ holds for some $k(0<k \leq N)$, then, from the definition of $\bar{I}_{k-1}$,

$$
\left(x_{k}, p_{k}, x_{k-1}, u_{k-1}, p_{k-1}, y_{k-1}\right) \in \mathcal{V}_{\mathbf{R}}\left(\bar{I}_{k-1}\right)
$$

holds with $y_{k-1}=1 / \bar{d}_{k-1}\left(x_{k-1}, u_{k-1}\right)$. By taking the projections of $\mathcal{V}_{\mathbf{R}}\left(\bar{I}_{k-1}\right)$ and by applying Lemma 1 to $\bar{I}_{k-1}$, we have

$$
\begin{aligned}
& \left(x_{k-1}, p_{k-1}\right) \in \pi_{\left(x_{k-1}, p_{k-1}\right)}\left(\mathcal{V}_{\mathbf{R}}\left(\bar{I}_{k-1}\right)\right) \subset \mathcal{V}_{\mathbf{R}}\left(I_{k-1}\right) \\
& \left(x_{k-1}, u_{k-1}\right) \in \pi_{\left(x_{k-1}, u_{k-1}\right)}\left(\mathcal{V}_{\mathbf{R}}\left(\bar{I}_{k-1}\right)\right) \subset \mathcal{V}_{\mathbf{R}}\left(K_{k-1}\right)
\end{aligned}
$$

which completes the proof by induction.

Remark 2: Although Algorithm 2 does not essentially resolve the double-exponential complexity in the computation of Gröbner bases, it can be more efficient than Algorithm 1 in practice, as shown in the example in Section VI. 


\section{Existence of Algebraic State Feedback Laws}

Note that a state feedback law consisting of algebraic functions is regarded as a point in $\overline{\mathbf{R}(x)}$. Therefore, a set of algebraic state feedback laws defined by a set of polynomials in $x$ and $u$ can be regarded as an affine variety defined by an ideal of $\mathbf{R}(x)[u]$ instead of $\mathbf{R}[x, u]$ in the previous section. To characterize affine varieties in $\overline{\mathbf{R}(x)}$, additional notions of commutative algebra and algebraic geometry are introduced for the polynomial ring $\mathbf{R}(x)[u]$. The same argument also applies to $\mathbf{R}(x)[p]$. The argument $x$ is often omitted for elements of $\mathbf{R}(x)[u]$ and $\mathbf{R}(x)[p]$ hereafter.

For an ideal $K \subset \mathbf{R}[x, u]$, the extension of $K$ to $\mathbf{R}(x)[u]$ is the ideal generated by $K$ over $\mathbf{R}(x)[u]$ and is denoted by $K^{e}$, i.e.,

$$
\begin{aligned}
K^{e}= & \left\{s_{1} G_{1}+\cdots+s_{\ell} G_{\ell}: s_{1}, \ldots, s_{\ell} \in \mathbf{R}(x)[u]\right. \\
& \left.G_{1}, \ldots, G_{\ell} \in K ; \ell \in \mathbf{N}\right\} .
\end{aligned}
$$

An ideal $J \in \mathbf{R}(x)[u]$ is called zero-dimensional if $\mathcal{V}(J) \subset \overline{\mathbf{R}(x)}^{m}$ is a finite set. Zero-dimensional ideals are characterized by the following lemma [15], [16].

Lemma 3: Ideal $J \subset \mathbf{R}(x)[u]$ is zero-dimensional if and only if there exists a nonzero polynomial $\psi_{i} \in \mathbf{R}(x)\left[u_{i}\right]$ for each $i=1, \ldots, m$ such that $J \cap \mathbf{R}(x)\left[u_{i}\right]=\left\langle\psi_{i}\right\rangle$.

The polynomial $\psi_{i} \in \mathbf{R}(x)\left[u_{i}\right]$ in Lemma 3 is called the minimal polynomial of $u_{i}$ with respect to $J$. Note that $J \cap \mathbf{R}(x)\left[u_{i}\right]=\left\langle\psi_{i}\right\rangle$ is an elimination ideal of $J$ and its generator $\psi_{i}$ can be computed by using a Gröbner basis.

If $a^{\nu} \in J$ for some integer $\nu$ always implies $a \in J$, then ideal $J$ is called radical. For an ideal $I \subset \mathbf{R}(x)[u]$, the set

$$
\sqrt{I}=\left\{a \in \mathbf{R}(x)[u]: a^{\nu} \in I \text { for some } \nu \in \mathbf{N}\right\}
$$

is a radical ideal and is called the radical of $I$. Obviously, $\mathcal{V}(I)=\mathcal{V}(\sqrt{I})$ holds. There is an algorithm for obtaining the radical of a zero-dimensional ideal [15], [16].

If a zero-dimensional ideal $J$ is also a radical ideal, the following lemma [17] gives the exact number of its generators and guarantees the nonsingularity of the Jacobian matrix defined by them, which is useful for further characterization of the elements of $\mathcal{V}(J)$ as implicit functions.

Lemma 4: If $J \subset \mathbf{R}(x)[u]$ is a zero-dimensional radical ideal, it has exactly $m$ generators $G_{1}, \ldots, G_{m} \in \mathbf{R}(x)[u]$, and $\operatorname{det}\left[\partial G_{i}\left(u^{*}\right) / \partial u_{j}\right] \neq 0$ for every $u^{*} \in \mathcal{V}(J)$.

This lemma is a straightforward extension of a similar lemma in [10] for a maximal ideal, using a characterization of zero-dimensional radical ideals [16], [18]. Finally, the Gröbner basis of a zero-dimensional radical ideal has a particular structure under a mild assumption [12], [15], which is useful for computing the generators in Lemma 4.

Now, the existence and uniqueness of costates and state feedback laws consisting of algebraic functions of the state are characterized under the following assumption.

Assumption 1: There exist sequences of states $\left(\bar{x}_{k}\right)_{k=0}^{N}$, costates $\left(\bar{p}_{k}\right)_{k=0}^{N}$, and inputs $\left(\bar{u}_{k}\right)_{k=0}^{N-1}$ such that the ELE hold and

$$
\frac{\partial^{2} H_{k}}{\partial u^{2}}+\left(\frac{\partial f_{k}}{\partial x}\right)^{\mathrm{T}} S_{k+1} \frac{\partial f_{k}}{\partial x}>0
$$

holds for $k=0, \ldots, N-1$, where $\left(S_{k}\right)_{k=1}^{N}$ is the solution to the following discrete-time Riccati equation:

$$
\begin{aligned}
S_{k}= & \frac{\partial^{2} H_{k}}{\partial x^{2}}+\left(\frac{\partial f_{k}}{\partial x}\right)^{\mathrm{T}} S_{k+1} \frac{\partial f_{k}}{\partial x} \\
& -\left(\frac{\partial^{2} H_{k}}{\partial x \partial u}+\left(\frac{\partial f_{k}}{\partial x}\right)^{\mathrm{T}} S_{k+1} \frac{\partial f_{k}}{\partial u}\right)^{-1} \\
& \times\left(\frac{\partial^{2} H_{k}}{\partial u^{2}}+\left(\frac{\partial f_{k}}{\partial u}\right)^{\mathrm{T}} S_{k+1} \frac{\partial f_{k}}{\partial u}\right)^{-1} \\
& \times\left(\frac{\partial^{2} H_{k}}{\partial u \partial x}+\left(\frac{\partial f_{k}}{\partial u}\right)^{\mathrm{T}} S_{k+1} \frac{\partial f_{k}}{\partial x}\right) \\
S_{N}= & \frac{\partial^{2} \varphi}{\partial x^{2}}\left(\bar{x}_{N}\right) .
\end{aligned}
$$

In (12) and (13), the arguments are $\left(\bar{x}_{k}, \bar{u}_{k}, \bar{p}_{k+1}\right)$ for partial derivatives of $H_{k}$ and $\left(\bar{x}_{k}, \bar{u}_{k}\right)$ for partial derivatives of $f_{k}$.

The ELE (2)-(5) together with (12) are the second-order sufficient conditions for local optimality [4].

Theorem 2: Suppose Assumption 1 holds and all $I_{k}^{e} \subset$ $\mathbf{R}\left(x_{k}\right)\left[p_{k}\right] \quad(k=0, \ldots, N)$ and $K_{k}^{e} \subset \mathbf{R}\left(x_{k}\right)\left[u_{k}\right]$ $(k=0, \ldots, N-1)$ obtained by Algorithm 2 are zerodimensional. Then, there exists a unique sequence of algebraic state feedback laws $u_{k}^{*} \in \mathcal{V}\left(K_{k}^{e}\right)(k=0, \ldots, N-1)$ that are defined on some neighborhoods of $x_{k}=\bar{x}_{k}$ and satisfy $u_{k}^{*}\left(\bar{x}_{k}\right)=\bar{u}_{k}$, and there exists a unique sequence of algebraic costates $p_{k}^{*} \in \mathcal{V}\left(I_{k}^{e}\right)(k=0, \ldots, N)$ that are defined on neighborhoods of $x_{k}=\bar{x}_{k}$ and satisfy $p_{k}^{*}\left(\bar{x}_{k}\right)=$ $\bar{p}_{k}$. Moreover, for any initial state in some neighborhood of $\bar{x}_{0}$, the closed-loop trajectory $\left(x_{k}^{C L}\right)_{k=0}^{N}$ given by $u_{k}^{*}\left(x_{k}^{C L}\right)$ gives a local optimal solution satisfying the ELE and (12) with the costates given by $p_{k}^{*}\left(x_{k}^{C L}\right)$.

Proof: For each $\ell=0, \ldots, N-1$, Assumption 1 implies that the sequences of states $\left(\bar{x}_{k}\right)_{k=\ell}^{N}$, costates $\left(\bar{p}_{k}\right)_{k=\ell}^{N}$, and inputs $\left(\bar{u}_{k}\right)_{k=\ell}^{N-1}$ satisfy the second-order sufficient conditions for local optimality for the optimal control problem with a horizon for $k=\ell, \ldots, N$. Then, by a classical result of sensitivity analysis [3], [19], there exists a unique set of differentiable functions $\left(x_{k}^{\ell}\left(x_{\ell}\right)\right)_{k=\ell}^{N},\left(p_{k}^{\ell}\left(x_{\ell}\right)\right)_{k=\ell}^{N}$, and $\left(u_{k}^{\ell}\left(x_{\ell}\right)\right)_{k=\ell}^{N-1}$ satisfying $x_{k}^{\ell}\left(\bar{x}_{\ell}\right)=\bar{x}_{k}, p_{k}^{\ell}\left(\bar{x}_{\ell}\right)=\bar{p}_{k}$, $u_{k}^{\ell}\left(\bar{x}_{\ell}\right)=\bar{u}_{k}$, and the second-order sufficient conditions for local optimality for any $x_{\ell}$ in some neighborhood of $\bar{x}_{\ell}$. The uniqueness of a local optimal solution implies that

$$
\begin{aligned}
& x_{k}^{\ell_{1}}\left(x_{\ell_{1}}^{\ell}\left(x_{\ell}\right)\right)=x_{k}^{\ell_{2}}\left(x_{\ell_{2}}^{\ell}\left(x_{\ell}\right)\right) \quad(k=\ell, \ldots, N), \\
& p_{k}^{\ell_{1}}\left(x_{\ell_{1}}^{\ell}\left(x_{\ell}\right)\right)=p_{k}^{\ell_{2}}\left(x_{\ell_{2}}^{\ell}\left(x_{\ell}\right)\right) \quad(k=\ell, \ldots, N), \\
& u_{k}^{\ell_{1}}\left(x_{\ell_{1}}^{\ell}\left(x_{\ell}\right)\right)=u_{k}^{\ell_{2}}\left(x_{\ell_{2}}^{\ell}\left(x_{\ell}\right)\right) \quad(k=\ell, \ldots, N-1),
\end{aligned}
$$

hold for any $\ell_{1}, \ell_{2} \in\{\ell, \ldots, k\}$ and for any $x_{\ell}$ in some neighborhood of $\bar{x}_{\ell}$. It is also obvious that $x_{\ell}^{\ell}\left(x_{\ell}\right)=x_{\ell}$ and $p_{\ell}^{\ell}\left(x_{\ell}\right)=p_{\ell}$ hold.

Now, define $u_{k}^{*}\left(x_{k}\right):=u_{k}^{k}\left(x_{k}\right)$ and $f_{k}^{C L}\left(x_{k}\right):=$ $f_{k}\left(x_{k}, u_{k}^{*}\left(x_{k}\right)\right)$ for $k=0, \ldots, N-1$. Then, $x_{k+1}^{k}\left(x_{k}\right)=$ $f_{k}^{C L}\left(x_{k}\right)$ holds for $k=0, \ldots, N-1$ and for any $x_{k}$ in some neighborhood of $\bar{x}_{k}$. This expression, together with 
(15) and $x_{0}^{0}\left(x_{0}\right)=x_{0}$, leads to

$$
\begin{aligned}
x_{k}^{0}\left(x_{0}\right) & =x_{k}^{k-1} \circ \cdots \circ x_{1}^{0}\left(x_{0}\right) \\
& =f_{k-1}^{C L} \circ \cdots \circ f_{0}^{C L}\left(x_{0}\right) \quad(k=0, \ldots, N) .
\end{aligned}
$$

That is, $\left(x_{k}^{0}\left(x_{0}\right)\right)_{k=0}^{N}$ is the closed-loop trajectory given by the state feedback laws $\left(u_{k}^{*}\left(x_{k}\right)\right)_{k=0}^{N-1}$, and $x_{k}^{C L}=x_{k}^{0}\left(x_{0}\right)$ holds. Similarly, we have

$$
\begin{aligned}
& u_{k}^{0}\left(x_{0}\right)=u_{k}^{k}\left(x_{k}^{0}\left(x_{0}\right)\right)=u_{k}^{*}\left(x_{k}^{C L}\right) \quad(k=0, \ldots, N-1), \\
& p_{k}^{0}\left(x_{0}\right)=p_{k}^{k}\left(x_{k}^{0}\left(x_{0}\right)\right)=p_{k}^{*}\left(x_{k}^{C L}\right) \quad(k=0, \ldots, N),
\end{aligned}
$$

where $p_{k}^{*}$ is defined as $p_{k}^{*}\left(x_{k}\right):=p_{k}^{k}\left(x_{k}\right)$. Therefore, the closed-loop trajectory $\left(x_{k}^{C L}\right)_{k=0}^{N}$ and corresponding inputs $\left(u_{k}^{*}\left(x_{k}^{C L}\right)\right)_{k=0}^{N-1}$ and costates $\left(p_{k}^{*}\left(x_{k}^{C L}\right)\right)_{k=0}^{N}$ are identical to the local optimal solution given by $\left(x_{k}^{0}\left(x_{0}\right)\right)_{k=0}^{N}$, $\left(p_{k}^{0}\left(x_{0}\right)\right)_{k=0}^{N}$, and $\left(u_{k}^{0}\left(x_{0}\right)\right)_{k=0}^{N-1}$, which satisfy the secondorder sufficient conditions for local optimality, namely, the ELE and (12).

Finally, it remains to show that the state feedback law $u_{k}^{*}\left(x_{k}\right)$ and the costate $p_{k}^{*}\left(x_{k}\right)$ are algebraic functions and belong to $\mathcal{V}\left(K_{k}^{e}\right)$ and $\mathcal{V}\left(I_{k}^{e}\right)$, respectively. For any $x_{k}$ in some neighborhood of $\bar{x}_{k}, x_{k}, p_{k}^{*}\left(x_{k}\right)$, and $u_{k}^{*}\left(x_{k}\right)$ belong to a local optimal solution satisfying the ELE. Therefore, Theorem 1 implies that $\left(x_{k}, p_{k}^{*}\left(x_{k}\right)\right) \in \mathcal{V}_{\mathbf{R}}\left(I_{k}\right)(k=$ $0, \ldots, N)$ and $\left(x_{k}, u_{k}^{*}\left(x_{k}\right)\right) \in \mathcal{V}_{\mathbf{R}}\left(K_{k}\right)(k=0, \ldots, N-1)$ hold. Since $I_{k}^{e}$ and $K_{k}^{e}$ are zero-dimensional, Lemma 3 implies the existence of the minimal polynomial of each $u_{k i}$ and $p_{k i}$ with respect to $K_{k}^{e}$ and $I_{k}^{e}$, respectively. Let $\psi_{k i}$ be the minimal polynomial of $u_{k i}(i=1, \cdots, m)$ with respect to $K_{k}^{e}$. By Remark $1, \psi_{k i}$ can be chosen from $\mathbf{R}\left[x_{k}, u_{k i}\right]$ so that $\psi_{k i} \in K_{k}$ also holds. Then, for any $x_{k}$ in the neighborhood of $\bar{x}_{k},\left(x_{k}, u_{k}^{*}\left(x_{k}\right)\right) \in \mathcal{V}_{\mathbf{R}}\left(K_{k}\right)$ implies that $\psi_{k i}\left(x_{k}, u_{k i}^{*}\left(x_{k}\right)\right)=0$. That is, every component $u_{k i}^{*}$ is a root of a polynomial $\psi_{k i} \in \mathbf{R}\left[x_{k}, u_{k i}\right]$, and, equivalently, is an algebraic function. Moreover, $\left(x_{k}, u_{k}^{*}\left(x_{k}\right)\right) \in \mathcal{V}_{\mathbf{R}}\left(K_{k}\right)$ also implies that $u_{k}^{*}$ is an element of $\overline{\mathbf{R}\left(x_{k}\right)}$ such that every polynomial in $K_{k}$ vanishes at $u_{k}^{*}$ and, therefore, every polynomial in $K_{k}^{e}$ also vanishes at $u_{k}^{*}$, which means $u_{k}^{*} \in \mathcal{V}\left(K_{k}^{e}\right)$. The same argument also applies to show $p_{k}^{*} \in \mathcal{V}\left(I_{k}^{e}\right)$.

If the assumptions in Theorem 2 are satisfied, Lemma 4 guarantees that $\sqrt{K_{k}^{e}} \in \mathbf{R}\left(x_{k}\right)\left[u_{k}\right](k=0, \ldots, N-$ $1)$ have exactly $m$ generators as $\sqrt{K_{k}^{e}}=\left\langle\bar{G}_{k}^{e}\right\rangle$, where $\bar{G}_{k}^{e} \in \mathbf{R}\left(x_{k}\right)\left[u_{k}\right]^{m}$. Lemma 4 also guarantees that $\partial \bar{G}_{k}^{e}\left(x_{k}, u_{k}^{*}\right) / \partial u \in{\overline{\mathbf{R}\left(x_{k}\right)}}^{m \times m}$ is nonsingular at each $u_{k}^{*} \in$ $\mathcal{V}\left(K_{k}^{e}\right)=\mathcal{V}\left(\sqrt{K_{k}^{e}}\right)$. Then, for $x_{k}$ in an open and dense subset of $\mathbf{R}^{n}, \partial \bar{G}_{k}^{e}\left(x_{k}, u_{k}^{*}\left(x_{k}\right)\right) / \partial u \in \mathbf{R}^{m \times m}$ is nonsingular. Therefore, Newton's method or the continuation method is applicable to $\bar{G}_{k}^{e}\left(x_{k}, u_{k}\right)=0$ to find $u_{k}^{*}\left(x_{k}\right) \in \mathbf{R}^{m}$ for $x_{k} \in \mathbf{R}^{n}$. The same argument also applies to $\sqrt{I_{k}^{e}}$ and $p_{k}$.

Alternatively, one can also use minimal polynomials of $u_{k i}(i=1, \ldots, m)$ to calculate $u_{k}^{*}\left(x_{k}\right)$. For example, if $K_{k}^{e}$ is zero-dimensional, its radical $\sqrt{K_{k}^{e}}$ is also zerodimensional and, by Lemma 3 , contains minimal polynomials $\psi_{k i} \in \mathbf{R}(x)\left[u_{k i}\right]$ of $u_{k i}(i=1, \ldots, m)$ such that $\sqrt{K_{k}^{e}} \cap \mathbf{R}(x)\left[u_{k i}\right]=\left\langle\psi_{k i}\right\rangle$ holds. Therefore, the $i$ th component of $u_{k}^{*} \in \mathcal{V}\left(K_{k}^{e}\right)$ is a root of $\psi_{k i}$, and the value of $u_{k i}^{*}\left(x_{k}\right) \in \mathbf{R}$ for some $x_{k} \in \mathbf{R}^{n}$ can be found by solving the univariate algebraic equation $\psi_{k i}\left(u_{k i}\right)=0$. In particular, the continuation method can also be used. Note that each component $u_{k i}^{*}\left(x_{k}\right)$ can be computed independently of other components.

\section{EXAMPLE}

Consider the following single-input two-dimensional system:

$$
x_{k+1}=\left[\begin{array}{c}
x_{k 2} \\
-x_{k 1}+\frac{u_{k}}{1+x_{k 1}}
\end{array}\right],
$$

together with the performance index

$$
J=\frac{1}{2}\left\|x_{4}\right\|^{2}+\sum_{k=0}^{3} \frac{1}{2}\left(\left\|x_{k}\right\|^{2}+\left\|u_{k}\right\|^{2}\right),
$$

for which the recursive elimination method (Algorithm 2 ) in Section IV is applied. It is obvious that the trivial solution to the ELE, $u_{k}=0(k=0, \ldots, N-1),\left(x_{k}, p_{k}\right)=$ $(0,0)(k=0, \ldots, N)$, satisfies the second-order sufficient conditions for local optimality because the linearization around $\left(x_{k}, u_{k}\right)=(0,0)$ gives a standard LQ control problem with positive definite weights for the state and input. Consequently, Assumption 1 in Theorem 2 holds.

Algorithm 2 yields a sequence of ideals $I_{k} \subset \mathbf{R}\left[x_{k}, p_{k}\right]$ as follows:

$$
\begin{aligned}
I_{4}= & \left\langle p_{41}-x_{41}, p_{42}-x_{42}\right\rangle, \\
I_{3}= & \left\langle\left(x_{31}^{4}+4 x_{31}^{3}+8 x_{31}^{2}+8 x_{31}+4\right) p_{31}-2 x_{31}^{5}\right. \\
& \left.-8 x_{31}^{4}-16 x_{31}^{3}-15 x_{31}^{2}-6 x_{31}, p_{32}-2 x_{32}\right\rangle, \\
I_{2}= & \left\langle\left(x_{21}^{4}+4 x_{21}^{3}+10 x_{21}^{2}+12 x_{21}+9\right) p_{21}-\cdots,\right. \\
& \left.\left(x_{22}^{4}+4 x_{22}^{3}+8 x_{22}^{2}+8 x_{22}+4\right) p_{22}-\cdots\right\rangle .
\end{aligned}
$$

Ideals $I_{1}$ and $I_{0}$ are omitted here due to the limitation of space. Ideal $I_{1}$ is generated by two polynomials: one is fifth degree in $p_{11}$ and the other is first degree in $p_{12}$. Ideal $I_{0}$ is also generated by two polynomials: one is fifth degree in $p_{01}$ and the other is fifth degree in $p_{02}$. In this example, every ideal $I_{k}$ is generated by two polynomials, one in $\mathbf{R}\left[x_{k}, p_{k 1}\right]$ and the other in $\mathbf{R}\left[x_{k}, p_{k 2}\right]$. Therefore, Lemma 3 implies that every $I_{k}^{e} \subset \mathbf{R}\left(x_{k}\right)\left[p_{k}\right]$ is zero-dimensional and is generated by the same polynomials as $I_{k}$. All $I_{k}^{e}$ are also radical because their generators are square-free [12]. Then, the costate $p_{k}^{*}$ can be obtained explicitly by Theorem 2 for $k=2,3,4$.

Algorithm 2 also yields a sequence of ideals $K_{k} \subset$ $\mathbf{R}\left[x_{k}, u_{k}\right]$ as follows:

$$
\begin{aligned}
& K_{3}=\left\langle\left(x_{31}^{2}+2 x_{31}+2\right) u_{31}-x_{31}^{2}-x_{31}\right\rangle, \\
& K_{2}=\left\langle\left(x_{21}^{2}+2 x_{21}+3\right) u_{21}-2 x_{21}^{2}-2 x_{21}\right\rangle, \\
& K_{1}=\left\langle\left(x_{11}^{2}+2 x_{11}+4\right) u_{11}^{5}+\cdots\right\rangle, \\
& K_{0}=\left\langle\left(x_{01}^{2}+2 x_{01}+5\right) u_{01}^{5}+\cdots\right\rangle .
\end{aligned}
$$

In this case, every ideal $K_{k}$ is generated by a single polynomial in $\mathbf{R}\left[x_{k}, u_{k}\right]$. Therefore, $K_{k}^{e} \subset \mathbf{R}\left(x_{k}\right)\left[u_{k}\right]$ is also generated by the same polynomial and, according to Lemma 3, is zero-dimensional. Moreover, every generator of $K_{k}$ is square-free, which implies that $K_{k}^{e}$ is also radical. 
Then, the optimal state feedback laws $u_{k}^{*}\left(x_{k}\right)$ for $k=$ 2,3 can be obtained explicitly as roots of $K_{2}^{e}$ and $K_{3}^{e}$, respectively. For $k=0,1,2$, since the degrees of the generators of $K_{k}^{e}$ are greater than or equal to 5 , the optimal state feedback laws cannot be obtained explicitly. However, these generators give exact algebraic equations that determine $u_{k}^{*}\left(x_{k}\right)$, which can be solved by the method described in the previous section. For example, the function $u_{0}^{*}\left(x_{0}\right)$ is shown in Fig. 1.

In this example, Algorithm 2 and Newton's method are implemented for the symbolic computation of the ideals and for the numerical computation of the state feedback law, respectively, using Mathematica on a PC (CPU: Intel Core i7-3520M 2.90 GHz, RAM: $7.88 \mathrm{~GB}$ ). The computational time for Algorithm 2 to obtain the sequences of ideals $I_{k}$ and $K_{k}$ is $1.0 \mathrm{~s}$. On the other hand, the computational time for obtaining the corresponding ideals $I_{k}^{E L E}$ and $K_{k}^{E L E}$ in Algorithm 1 is $77.9 \mathrm{~s}$. Therefore, Algorithm 2 is computationally more efficient than the simple application of the elimination method to all the ELE.

The maximum computational time required to numerically obtain each control input in Fig. 1 is $0.73 \mathrm{~ms}$ with an average time of $0.67 \mathrm{~ms}$. On the other hand, when Newton's method is used to solve all the ELE, the maximum computational time required to obtain the same control input is $2.5 \mathrm{~ms}$ and the average time is $1.8 \mathrm{~ms}$. Therefore, the proposed method is computationally more efficient than solving all the ELE numerically when realtime computation of the state feedback law is necessary at each time.

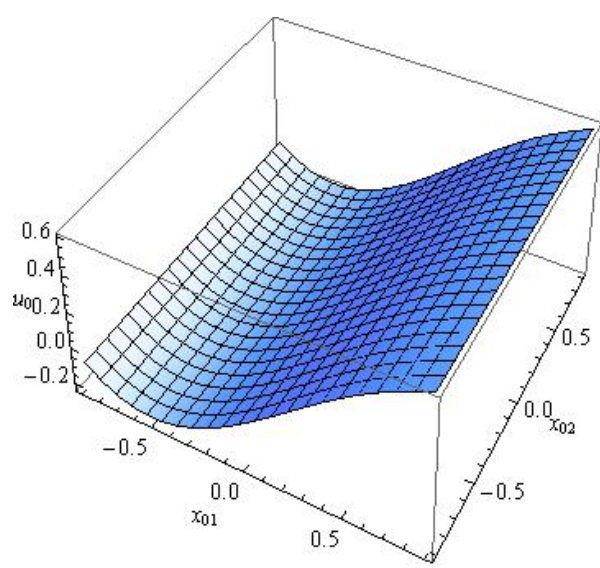

Fig. 1. Optimal state feedback law $u_{0}^{*}\left(x_{0}\right)$.

\section{CONCLUSION}

In this technical note, a recursive elimination method has been proposed for solving the ELE in FHOCPs of discrete-time rational systems. At the expense of restricting the system to consist of rational functions, some tools from commutative algebra and algebraic geometry are applied. Instead of the numerical solution or symbolic manipulation of all the ELE as a whole, sequences of algebraic equations for the control input and costate at each time are constructed backward, starting from the terminal condition in the ELE. There is no approximation involved in the construction of the algebraic equations, in contrast to most conventional approaches to nonlinear optimal control problems. Sufficient conditions were given for the existence and uniqueness of locally optimal state feedback laws in the form of algebraic functions. It was shown in the example that the proposed method can be computationally more efficient than dealing with all the ELE as a whole.

\section{REFERENCES}

[1] B. D. O. Anderson and J. B. Moore, Optimal Control. Englewood Cliffs, NJ: Prentice Hall, 1989.

[2] T. Ohtsuka, "A recursive elimination method for optimal control of discrete-time polynomial systems," in Preprints of 4 th IFAC Nonlinear Model Predictive Control Conference, Noordwijkerhout, 2012, pp. 916-919.

[3] M. Fukushima, Fundamentals of Nonlinear Optimization. Tokyo: Asakura Publishing, 2001, (in Japanese).

[4] A. E. Bryson, Jr. and Y.-C. Ho, Applied Optimal Control. Washington, DC: Hemisphere, 1975.

[5] U. Walther, T. T. Georgiou, and A. Tannenbaum, "On the computation of switching surfaces in optimal control: A Gröbner basis approach," IEEE Transactions on Automatic Control, vol. 46, no. 4, pp. 534-540, 2001.

[6] I. A. Fotiou, P. Rostalski, P. A. Parrilo, and M. Morari, "Parametric optimization and optimal control using algebraic geometry methods," Inernational Journal of Control, vol. 79, no. 11, pp. $1340-1358,2006$.

[7] H. Ichihara, "Optimal control for polynomial systems using matrix sum of squares relaxations," IEEE Transactions on Automatic Control, vol. 54, no. 5, pp. 1048-1053, 2009.

[8] P. Rostalski, I. A. Fotiou, D. J. Bates, A. G. Beccuti, and M. Morari, "Numerical algebraic geometry for optimal control applications," SIAM Journal on Optimization, vol. 21, no. 2, pp. 417-437, 2011.

[9] H. Iwane, A. Kira, and H. Anai, "Construction of explicit optimal value functions by a symbolic-numeric cylindrical algebraic decomposition," in Computer Algebra in Scientific Computing, ser. Lecture Notes in Computer Science, V. P. Gerdt, W. Koepf, E. W. Mayr, and E. V. Vorozhtsov, Eds. Berlin: SpringerVerlag, 2011, vol. 6885 , pp. 239-250.

[10] T. Ohtsuka, "Solutions to the Hamilton-Jacobi equation with algebraic gradients," IEEE Transactions on Automatic Control, vol. 56 , no. 8 , pp. $1874-1885,2011$.

[11] D. Něsić, I. M. Y. Mareels, T. Glad, and M. Jirstrand, "The Gröbner basis method in control design: An overview," in Proceedings of the 15th IFAC World Congress, Barcelona, 2002, pp. $1052-1057$.

[12] T. Becker and V. Weispfenning, Gröbner Bases. New York, NY: Springer-Verlag, 1993.

[13] D. Cox, J. Little, and D. O'Shea, Ideals, Varieties, and Algorithms, 3rd ed. New York, NY: Springer, 2007.

[14] D. T. Huynh, "A superexponential lower bound for Gröbner bases and Church-Rosser commutative Thue systems," Information and Control, vol. 68, pp. 196-206, 1986.

[15] M. Kreuzer and L. Robbiano, Computational Commutative Algebra. Berlin: Springer-Verlag, 2000, vol. 1.

[16] D. Cox, J. Little, and D. O'Shea, Using Algebraic Geometry, 2nd ed. New York, NY: Springer, 2005.

[17] Y. Kawano and T. Ohtsuka, "Algebraic solutions to the Hamilton-Jacobi equation with the time-varying Hamiltonian," SICE Journal of Control, Measurement, and System Integration, vol. 6, no. 1, pp. 28-37, 2013.

[18] W. Vasconcelos, Computational Methods in Commutative Algebra and Algebraic Geometry, ser. Algorithms and Computation in Mathematics. Berlin: Springer-Verlag, 2004, vol. 2.

[19] A. V. Fiacco, Introduction to Sensitivity and Stability Analysis in Nonlinear Programming. New York, NY: Academic Press, 1983. 